\title{
Multiple pyogenic brain abscesses: an analysis of 21 patients
}

\author{
ALI SYED BASIT, * BHATIA RAVI, $\dagger$ A K BANERJI, $\uparrow$ P N TANDON $\dagger$ \\ From Dammam Central Hospital, ${ }^{*}$ Dammam, K.S.A., All India Institute of Medical Sciences, $\dagger$ New Delhi, \\ India
}

SUMMARY Multiple pyogenic brain abscesses constituted $11.7 \%$ (21 patients) of a series of 179 consecutive patients with brain abscess treated from 1976 to 1984 . The commonest cause of these abscesses was congenital cyanotic heart disease $(38 \%)$, followed by middle ear infection (19\%). Two thirds of these patients were in the first two decades of life. The clinical presentation was similar to brain abscesses in general, there being no clinical features suggestive of multiple lesions. In $85 \%$ the abscesses were supratentorial. Infratentorial abscesses alone or in association with supratentorial abscesses were invariably otogenic in origin. Aspiration of the largest abscesses supplemented with appropriate antibiotics was the treatment of choice in 16 patients; secondary excision of the largest abscesses was required in six patients. Primary excision was performed in three patients when the abscesses were in close proximity to each other. Two patients were managed with antibiotics alone. The overall mortality was $23 \cdot 8 \%$.

A very high mortality ranging $50-100 \%^{1-8}$ has been reported for multiple brain abscesses. There have been few reports of successful management of these patients in recent years and most reports cite only single or at most two cases. ${ }^{9-16} \mathrm{~A}$ review of 21 patients with multiple brain abscesses treated from 1976 to 1984 in the Department of Neurosurgery, All India Institute of Medical Sciences is reported, to highlight that prompt diagnosis, appropriate therapy and careful monitoring can provide vastly improved results.

\section{Material and methods}

In a consecutive series of 179 patients of pyogenic brain abscess treated over a nine year period, there were 21 patients $(11.7 \%)$ with multiple abscesses. Diagnosis was made on the basis of contrast enhanced CT in 19 patients. Diagnosis was established at surgery in two patients whose pre-operative investigation was an angiogram or a ventriculogram. On contrast enhanced CT scans the abscesses presented as a central low density core (0-15 Hounsfield Units) surrounded by a well defined regular enhancing ring (35-50 Hounsfield Units), producing a mass effect. Varying degree of surrounding low attenuating oedema was a common finding. This CT appearance was distinguished from other causes of ring lesions on the basis of clinical history..$^{17}$

Address for reprint requests: Dr Syed Basit Ali, Department of Neurosurgery, Dammam Central Hospital, Dammam 3196, Saudi Arabia.

Received 30 September 1988.

Accepted 1 January 1989
Abscesses were considered multiple when there were two or more abscesses at a distance from each other clearly separated by brain parenchyma. Care was taken to distinguish multiplicity from multiloculation on the CT image by studying the details of serial sections. Patients with single intracerebral abscess along with subdural empyema were not considered as cases of multiple abscesses.

Age and sex distribution The youngest patient was 4 years old and the oldest 35 years. The mean age was 14 years with a male preponderance (3:1). Three fourths of the patients were in the first two decades of life (table 1).

Aetiology The commonest association was with congenital cyanotic heart disease (eight patients). Chronic middle ear infection was responsible for brain abscesses in four patients. In three patients abscesses were related to head injury; two of these had a compound head injury and one a closed injury. In two patients multiple abscesses were discovered by CT who were being treated for pyogenic meningitis. In two patients, the precise cause could not be ascertained. In the remaining two patients, one was thoracogenic in origin and the other followed osteomyelitis of the calvarium.

Clinical features Clinical features of raised intracranial

Table 1 Age and sex distribution

\begin{tabular}{lllcl}
\hline Age (years) & Male & Female & Total & Died \\
\hline$\leqslant 10$ & 7 & 2 & 9 & 2 \\
$11-20$ & 5 & 2 & 7 & 2 \\
$21-30$ & 2 & 1 & 3 & 1 \\
$31-40$ & 2 & - & 2 & $\frac{1}{5}$ \\
Total & 16 & 5 & 21 & \\
\hline
\end{tabular}


Table 2 Conscious level on admission

\begin{tabular}{lrrr}
\hline Level of consciousness & No & Died & $\%$ \\
\hline I-Conscious and oriented & 11 & 1 & 9 \\
II-Drowsy and disoriented but responding & 9 & 3 & 33 \\
$\quad$ to commands & 1 & 1 & 100 \\
III-Responding only to painful stimuli & 21 & 5 & \\
Total &
\end{tabular}

pressure characterised by headache, vomiting and papilloedema were present in the majority (15 patients). Only 14 patients had a definite history of fever prior to admission. Focal or generalised seizures were present in nine patients and one of them had lapsed into status epilepticus. Motor deficit was recorded in 10 patients. In a third of the patients signs of meningeal irritation were present on admission. Eleven patients were fully conscious and oriented on admission (table 2). None of the cases had features which suggested multiple brain lesions. The duration of symptoms ranged from two weeks to six months but two thirds of the patients had been symptomatic for less than a month. The number of abscesses ranged from two to nine. Two abscesses were present in eight patients, three abscesses in seven patients, four abscesses in three patients, six abscesses in two patients and the remaining patient had nine abscesses. The location of abscesses is shown in table 3 .

Microbial analysis A microbial analysis of pus was available in 19 cases, as two cases were managed by antibiotics alone. Pure culture of aerobic organisms were found in seven cases. In three cases both aerobic and anaerobic organisms were isolated. Anaerobic organisms alone were isolated in only one case. In four cases culture revealed multiple organisms. In eight cases the culture was sterile.

Treatment (table 4) Free hand or stereotactic aspiration of the largest abscesses was performed in 16 cases. In six of them secondary excision of the largest abscess along with adjacent lesion was carried out. In ten cases aspiration was performed alone; six of them required repeated aspirations. In both groups while the largest abscesses were thus treated, other smaller abscesses were allowed to resolve under antibiotic therapy.

Two cases with smaller abscesses diagnosed on contrast enhanced CT were treated by antibiotics alone.

Primary excision of abscesses was carried out in three cases and in all these the abscesses were in one hemisphere. Two of these cases were operated upon before the CT scanner was available. The third case was of post traumatic abscesses who required excision twice for recurrence. All cases were followed by serial CT scanning.

All cases were given parenteral crystalline penicillin ( 2

Table 3 Location of abscesses

\begin{tabular}{|c|c|c|c|}
\hline & & No & Died \\
\hline $\begin{array}{l}\text { Supratentorial } \\
\text { Right hemisphere } \\
\text { Left hemisphere } \\
\text { Both hemispheres }\end{array}$ & $\begin{array}{l}5 \\
5 \\
8\end{array}$ & 18 & 4 \\
\hline $\begin{array}{l}\text { Both supra and Infratentorial } \\
\text { Infratentorial }\end{array}$ & & $\begin{array}{l}2 \\
1\end{array}$ & 1 \\
\hline
\end{tabular}

Table 4 Mode of therapy

\begin{tabular}{llll}
\hline Treatment & No & Died \\
\hline Aspiration only & 4 & 10 & \\
$\quad$ Once & 6 & & 2 \\
$\quad$ Repeated & & 6 & 3 \\
Secondary excision & & 3 & - \\
Primary excision & 2 & - \\
Only antibiotics & & 2 & \\
\hline
\end{tabular}

mega units 2 hourly) and chloramphenicol ( 1 g 6 hourly). The antibiotics were altered according to the microbial culture and sensitivity report. Metronidazole ( $500 \mathrm{mg} 8$ hourly) was used in cases in which anaerobic organisms were isolated. All cases were given anti-convulsant drugs (diphenyl hydantoin in a dose of $5 \mathrm{mg} / \mathrm{kg} /$ day and/or phenobarbitone $3 \mathrm{mg} / \mathrm{kg} /$ day). Steroids were used only for a short period in patients who were deteriorating despite antibiotics and decongestive therapy. Parenteral dexamethesone was used in the dosage of $4 \mathrm{mg}$ six hourly for five days then tapered rapidly.

It was observed that while oedema and corresponding midline shifts responded readily to therapy, the ring lesion took a longer time to resolve. Antibiotics were continued for six weeks after complete resolution of all ring lesions.

\section{Results}

Five cases died (tables 1 to 4). The major cause of $\frac{}{\sigma} \vec{z}$ death in these cases was attributed to septicaemia, $\varnothing 2$ meningitis, ventriculitis. One case in addition had status epilepticus.

Surprisingly, cases with congenital cyanotic heart. disease did better; only one of the eight cases died. There were two deaths among four cases of otogenic origin. There was one death in the post-traumatic group. The patient with osteomyelitis of the calvarium was well for six weeks after secondary excision of abscesses and the osteomyelitic bone. He was readmitted with rupture of a small juxtaventricular abscess into the ventricle and died of ventriculitis.

There was no mortality amongst those patients who required aspiration of their abscess only once (table 4).

No significant correlation was found between organism isolated and the mortality.

With one exception all those who died had abscesses in both hemispheres. One of these patients had both supra and infratentorial abscesses (table 3).

Follow up The follow up was one to seven years, except for one patient who has been followed for only eight weeks. Five patients had follow-up between five and seven years, seven between three and five years and three between one and three years. Among the former group two patients continued to have seizures. One patient started having seizures after three years. There was only one patient with minimal residual hemiparesis and the rest were well without focal neurological deficit. 
Discussion

In various reported series of brain abscesses, the incidence of multiple brain abscesses has been reported to vary from $4 \%$ to $20 \%,,^{125-717-22}$ as compared with $11.7 \%$ in the present series.

Before the introduction of CT, diagnosis of multiple brain abscesses was mostly at necropsy, sometimes during surgery if the other abscess/abscesses were in the neighbourhood, and occasionally on conventional neuroradiography. With the availability of CT an abscess smaller than $1 \mathrm{~cm}$ in diameter can be visualised. ${ }^{23}$ However, these still need to be distinguished from metastasis, granulomas, neoplasms with partly necrotic centres, recent infarcts, contusions, haematomas and even acute demyelinating processes. ${ }^{92324}$

The commonest cause of multiple abscesses in our series was congenital cyanotic heart disease. In contrast the commonest cause for a brain abscess in general in most reported series is middle ear infection, ${ }^{13-6818192225-27}$ the notable exception being the report by $\mathrm{Hirsch}^{28}$ who found congenital cyanotic heart disease as the commonest cause.

In the majority of our patients (18 patients) the abscesses were supratentorial. In two cases the abscesses were present both above and below the tentorium and in one all abscesses were below the tentorium.

The clinical presentation of multiple brain abscesses was essentially similar to other abscesses, ${ }^{35781819212225-34}$ there being no clinical evidence of multiplicity in any case.

Microbial examination revealed a variety of aerobic and anaerobic pyogenic organisms. Four out of 11 positive cultures revealed anaerobic organisms. In a significant number of patients (eight) the culture grew no organisms.

The majority of patients (16) were treated by aspiration of the larger abscesses, either once or repeatedly. Six of these required secondary excision. In three patients where the other abscesses were located in close proximity, primary excision was carried out. In two patients with multiple small abscesses, treatment was successful with antibiotics.

A very high mortality ranging from $50-100 \%$ has been reported for multiple brain abscesses. ${ }^{1-8}$ Carey $e t$ $a l,{ }^{\prime}$ Garfield $^{2}$ and McClelland et $a l^{16}$ reported $100 \%$ mortality and Kiser and Kendig' lost $85 \%$ of their patients. The overall mortality in our series was $23.8 \%$. Precise diagnosis with CT, aspiration of the larger abscess to relieve raised pressure, appropriate antibiotic therapy based on the results of culture and sensitivity, careful monitoring with repeated CT, all contributed to achieve these results.
References

1 Carey ME, Chou SN, French LA. Experience with brain abscesses. J Neurosurg 1972;36:1-9.

2 Garfield J. Management of supratentorial intracranial abscess-a review of 200 cases. Br Med J 1969;2:7-11.

3 Bhatia R. Brain abscess. Progress in Clinical Medicine in India. Ahuja MMS, ed. India, Arnold-Heinemann, 1984:634.

4 Brewer NS, MacCarty CS, Wellman WE. Brain abscesses. A review of recent experience. Ann Internal Med 1975;82:571-6.

5 Kiser JL, Kendig JH. Intracranial suppuration: a review of 139 consecutive cases. Electron microscope observation in three. $J$ Neurosurg 1963;20:494-511.

6 McClelland CJ, Craig BF, Cockard HA. Brain abscesses in Northern Ireland, a $\mathbf{3 0}$ year community review. J Neurol Neurosurg Psychiatry 1978;41:1043.

7 Morgan H, Wood MW, Murphy F. Experience with 88 consecutive cases of brain abscesses. J Neurosurg 1973;38:698.

8 Nulsen H, Glydensted C, Harmsen A. Cerebral abscesses. Aetiology, Pathogenesis, Symptoms, Diagnosis and treatment. A review of 200 cases from 1935-76. Acta Neurol Scandinav 1982;65:609-22.

9 Barsoum AH, Lewis HC, Cannillo KL. Non-operative treatment of multiple brain abscesses. Surg Neurol 1981;16(A):283-7.

10 Burke LP, Ho SH, Cerullo LJ, Kim KS, Harter DH. Multiple brain abscesses. Surg Neurol 1981;16:452-54.

11 George B. Antibiotic therapy for multiple brain abscess (letter). $J$ Neurosurg 1981;55:153-4.

12 George B, Roux F, Pillon M, Thurel C, George C. Relevance of antibiotics in the treatment of brain abscesses. Report of a case with eight simultaneous brain abscesses treated and cured medically. Acta Neurochir 1979;47:285-91.

13 Hubschmann OR, Weisborot FJ, Smith LG. Multiple Streptococcal brain abscesses successfully treated by craniotomy and needle aspiration. Surg Neurol 1982;17:57-61.

14 Hughes RB, Fachnle ST, Simon JL. Multiple cerebral abscesses complicating hepato pulmonary amoebiasis. J Paediatrics 1975; 86:95.

15 Kobrine AI, Davis DO, Rizzoli HV. Multiple abscesses of the brain-Case report. J Neurosurg 1981;54:93.

16 Junichi Imamura, Yu'taka Watanabe. Multiple brain abscesses associated with a mycotic aneurysm of the left common carotid artery. J Neurosurg 1986;64:325-7.

17 Danziger A, Price H, Schecker MM. An analysis of 113 intracranial infections. Neuroadiology 1980;19:31-4.

18 Dharkar SR, Shadangi TM, Vaishya ND, Arora VK, Dharkar RS. Pyogenic brain abscess-experience with 87 cases. Neurology (India) 1978;26:126-30.

19 Dohrmann PJ, Elrick WL. Observations on Brain abscess: Review of 28 cases. Med J Austr 1982;2:81-3.

20 Jooma OV, Pennybacker JB, Tutton GK. Brain abscess: aspiration, drainage or excision? J Neurol Neurosurg Psychiatry 1951; 14:308-13.

21 Rosenblum ML, Hoff JT, Norman D, Weinsten PR, Pitts L. Decreased mortality from brain abscesses since advent of computed tomography. J Neurosurg 1978;49:658-68.

22 Yang SY. Brain abscess: a review of 400 cases. J Neurosurg 1981; 55:794-99.

23 Kaufman DM, Leeds NE. Computed Tomography (CT) in the diagnosis of intracranial abscesses. Brain abscess, subdural empyema and epidural empyema. Neurology 1977;27:1069-73.

24 Whelan MA, Hilal SK. Computed tomography as a guide in the diagnosis and follow up of brain abscesses. Neuroradiology 1980;135:663-71.

25 Bhatia R. Brain abscess in children. Indian J Pediatr 1983;50: 591-7.

26 Bhatia R, Tandon PN, Banerji AK. Brain abscess-An analysis of 
55 cases. International Surgery 1973;58:565-8.

27 Bellar AJ, Sahar A, Praiss I. Brain abscess-a review of 89 cases over a period of 30 years. J Neurol Neurosurg Psychiatry 1973; 36:757.

28 Hirsch JF, Raux FX, Sainte RC, Renier D, Purre KA. Brain abscess in childhood. A study of 34 cases treated by puncture and antibiotics. Childs Brain 1983;10:251-65.

29 Mathai KV. Intracranial space occupying lesions. Review of 2332 cases. Neurology (India) 1978;26:157-70.

30 Bhatia R, Tandon PN, Banerji AK, Prakash B. Brain abscess and congenital heart disease. Acta Neurochir 1976;33:233.
31 Clark DB. Brain abscess and congenital heart disease. Clinical Neurosurgery Proceedings of the Congress of Neurological Surgeons. In: San Juan Puerto Rico, ed. 1966, Baltimore: William \& Wilkin's Company, 1967:274.

32 Garfield J. Brain abscess and other suppurative lesions of nervous system. Hand Book of Clinical Neurology. Vinken PJ, Bruyn GW, eds. Amsterdam: vol. 33:107.

33 Kagawa M, Takeshita M, Yato S, Kitamura K. Brain abscess in congenital cyanotic heart disease. $J$ Neurosurg 1983;58:913-7.

34 Raimondi AJ, Matsumoto S, Miller RA. Brain abscess in children with congenital heart disease. J Neurosurg 1965;23:588-95.

\section{Raynaud's phenomenon}

Maurice Raynaud (1834-81) provided one of the finest legacies of descriptive medicine and clinically inferred physiology. ${ }^{12}$

“. . . local syncope is a condition perfectly compatible with health . . ordinarily females, sometimes without appreciable cause, one or more fingers becomes pale and cold all at once... It is the phenomenon known as 'dead finger'. The attack is indolent, the duration varies from a few minutes to many hours. The determining cause is often the impression of cold; . . sometimes even a simple mental emotion is enough. It appears completely exsanguine . . . sensibility becomes blunted, then annihilated; fingers . . . can be pinched and pricked with impunity; may loose sense of contact and yet be able to distinguish heat and cold. Their temperature is notably lowered; He asks is the loss of movement due to a temporary paralysis? "This is hardly possible if one considers that this syncopal state is often limited to a single digit, . . . It is more reasonable to admit that the diseased part no longer transmitting sensation to the brain, the brain itself loses temporarily, for want of excitant, the power of determining movements. " He continues by mentioning involvement of the toes and says the attack "is followed by a reaction, often very painful ... in more pronounced cases the asphyxial element predominates, pallor is replaced by a cyanotic colour ... skin had acquired a greater transparence than natural so as to allow subjacent tissues to be perceived; sometimes violet or slate coloured, even becoming black, ..."He distinguished the condition from "concussion or compression of a nerve," and noted "feebleness of the pulse at the commencement . . . and the patch of white produced by pressure . . . takes a considerable time before recovering colour . . which denotes an excessive slowness of the capillary circulation. . . painful numbness succeeds a sensation of burning and shooting ... Meanwhile cutaneous anaesthesia is complete and interferes with the prehension of small objects. The period of reaction . . . irritating tingling sensations . . . livid patches a vermilion colour shows itself at the margin; little by little it gains ground, chasing before it the bronzed colour ... at the periphery. Finally, a patch of deep red ... "These observations lead onto sections on diagnosis and the nature of the disease in which he clearly recognises its vascular basis and its origin in " $a$ disorder of the ganglionary system and the great sympathetic nerves of the neck described by M. Claude Bernard." Raynaud infers "a sort of painful cramp of the arteries, ... spasm of vessels irregular or intermittent".

On treatment he advises "envelopment off the limb in close fitting cotton wool and, opium to calm the atrocious pains of mortification of the extremities." Most importantly, Raynaud stressed the benign nature of the condition and that "it is not a true gangrene which is produced".

\section{References}

1 Raynaud M. On local asphyxia and symmetrical gangrene of the extremities. trans T Barlow, London, The New Sydenham Society, 1988; 99-101, 138-40;143-5;147-50.

2 Raynaud M. De l'asphyxie locale et de la gangrene symmetrique des extemities. Paris. Rignoux 1862. 